\title{
Review of Color Image Segmentation
}

\author{
Abderrahmane ELBALAOUI ${ }^{1}$ M.FAKIR ${ }^{2}$ \\ Faculty of Science and Technology/Informatics, \\ Beni-Mellal, Morocco.
}

\begin{abstract}
This paper provides a review of methods advanced in the past few years for segmentation of color images. After a brief definition of the segmentation, we outline the various existing techniques, classified according to their approaches. We have identified five that are based approaches contours, those relying on notion of region, structural approaches, those based on the form and then using those notions of graphs. For each of these approaches, we then explained and illustrated their most important methods. This review is not intended to be exhaustive and the classification of certain methods may be discussed since at the boundary between different approaches.
\end{abstract}

Keywords-Image segmentation; k-means; region and Boundary.

\section{INTRODUCTION}

Image segmentation is one of the fundamental and most studied problems in computer vision. Segmentation is a commonly used term for identifying differences between particularly interesting and uninteresting objects as well as distinguishing foreground from background content. Segmentation is to partition an image into homogeneous regions that is to say, a connected set of points in the image with common properties. This important step in the process and analysis of color images refers to the feature extraction using two approaches commonly known as: region approach and contour approach. We briefly give the differences between these two concepts:

1) The notion of "contour" is associated with the search for local discontinuities, transitions between different areas where certain parameters such as color, intensity, texture are not identical. But this notion of contour does not directly yield a segmentation of the image. Indeed, it is quite rare to get directly after treatment, outlines fully connected and it is then necessary to close the contours in order to then assimilate regions to areas within a closed line.

2) The concept of "region" is associated with clusters of connected pixels with common attributes (color, texture). These methods lead directly to a partition of the image where each pixel is assigned to a single region.

The paper is organized as following. Section 2 we focus on different methods of region, those based on the form, those based on a paradigm of regions, those using a structural approach, those using graph theory and finally those based on classification. Section 3 we describe the methods that we classified into five major themes that are operating on the outline approaches.

\author{
N.IDRISSI ${ }^{3}$, A.MARBOHA ${ }^{4}$ \\ Faculty of Science and Technology /Informatics, \\ Beni-Mellal, Morocco.
}

\section{REGION APPROACH}

Region approach seeks to combine pixel homogeneous regions. it is characterized by the extent of uniformity of the built-up areas in the image. These regions are constructed by evaluating the similarity between the pixels or between a pixel and those of the same region. Methods are distinguished by region growing division-fusion and classification.

\section{A. Segmentation analysis of spatial properties}

This first part is devoted to methods that consider a region as a set of connected pixels whose colors are close to each other. These methods operate by scanning the image in order to build and involve regions the color information for decision making. We can distinguish four approaches:

- Regions dividing [1].

- Region Growing [2].

- Region Merging [4].

- Split-and-merge [5].

1) Regions dividing

The segmentation approach by dividing regions is to divide the original image into homogeneous regions in terms of a given criterion.

This process is recursive and considers that the initial region corresponds to the image to be analyzed. If a region does not meet homogeneity predicate, it is divided into four sub-regions of equal size. Each sub-region is analyzed. The recursive algorithm stops when all regions meet the homogeneity predicate.

Because of the division into four regions, this method is more adapted to square images having a number of rows and columns equal to a power of two, and wherein the regions are rectangular in shape. On the other hand, this method tends to show the effects of blocks.
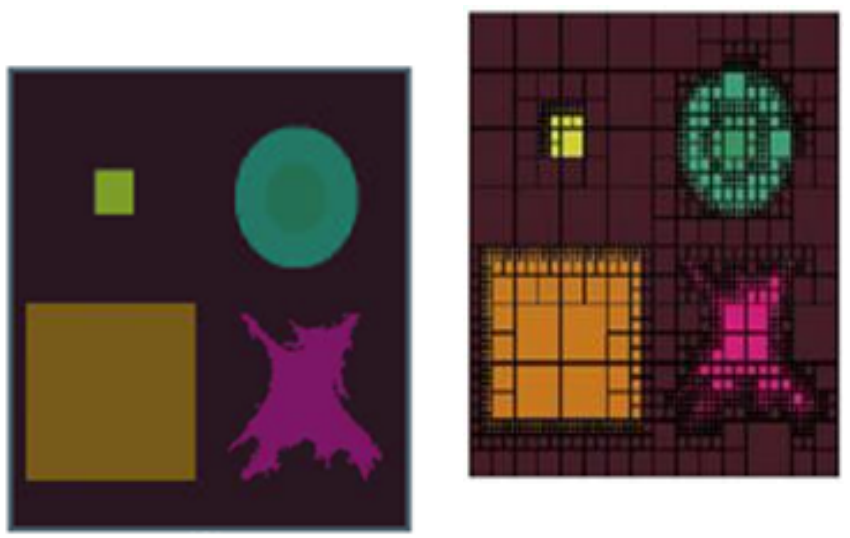
(1)

Fig. 1. (1) Original image, (2) Image segmented[1].

\section{2) Region Growing}

This type of segmentation is to grow regions by adding successively adjacent pixels that meet a criterion of homogeneity. Growth stops when all pixels have been processed.

The initial step is to select seed regions that generally correspond to a pixel. Then, regions are constructed by successively adding pixels that are related and that satisfy a similarity criterion color.

Most algorithms [2] growth regions are covered by a path of the image scanning from left to right and from top to bottom (fig.2).

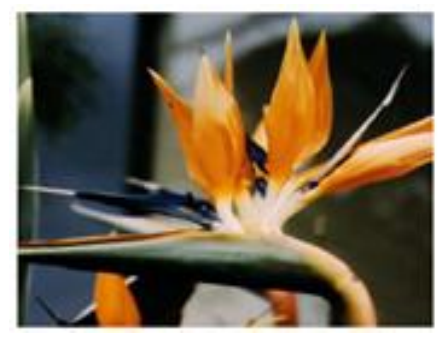

(1)

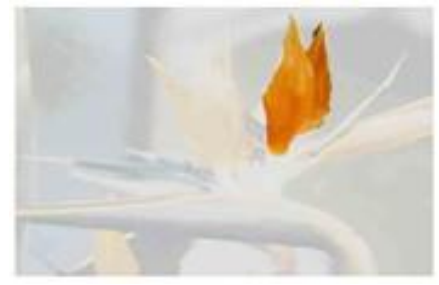

(4)

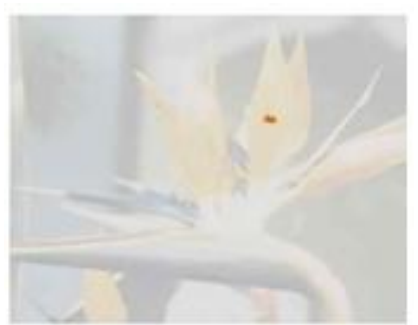

(2)

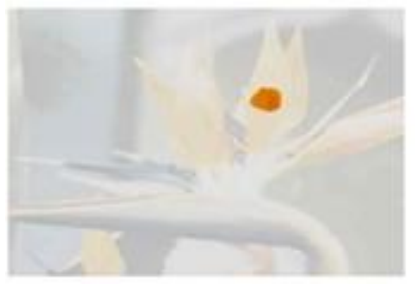

(3)
Fig. 2. (1) Original image, (2) Seed, (3) Growth, (4) region final [2].

The advantage of the growth areas is to preserve the shape of each region of the image.

However, poor selection of seeds or a choice of similarity criterion unsuitable phenomena can cause sub-segmentation or over-segmentation.

\section{3) Region Merging}

The region merging approach is mainly based on the analysis of an adjacency graph regions which analyzes a segmented image consists of a set of regions.

This is a data structure consisting of a non-oriented graph of which each node represents a region and each edge represents an adjacency between two regions.

Fig.3 (2) shows, as an example, the adjacency graph of regions obtained from the image in Fig.3 (1) .

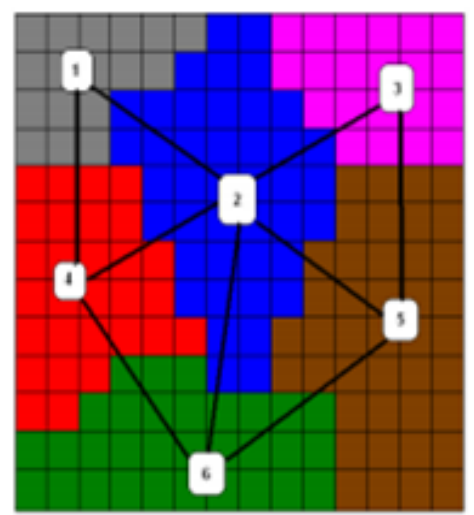

(1)

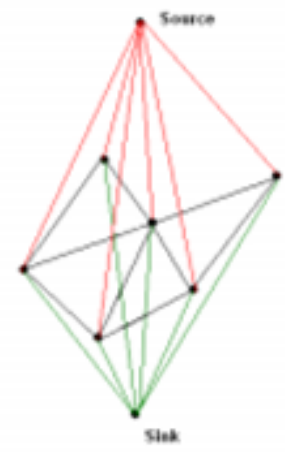

(2)
Fig. 3. (1) Original image, (2) Graph adjacency[4]

The process of merging two nodes connected by an edge provided they meet a criterion of fusion.

R. Schettini [3] proposes the use of a criterion taking into account the similarity of two color regions and spatial proximity. It introduces a similarity function based on a color distance weighted by the relative length of the common border of the two regions to merge.

J. Stawiaski [4] proposes a weighting each edge adjacency graph of the regions by half the distance between the two color regions corresponding to the nodes of the edge.

At each iteration, regions connected by the bridge which carries the minimum weight are merged. The edge weights are updated according to the number of pixels belonging to edges associated with their color and distance regions. With the weighting chosen, the algorithm tends to favor the adjacent regions of low surface. The fusion algorithm stops when previously determined number of iterations is reached or when the weights of the edges reach a limit value.

\section{4) Split-and-Merge}

Segmentation Split-and-Merge combines the previous two approaches. Firstly, the image is divided into homogeneous regions that meet global criteria and adjacent areas that meet local criteria are merged. We describe two data structures to perform this approach: the quadtree and the Voronoi diagram.

\section{a) quadtree}

V. Coutance [5] segmenting color images into regions by analyzing a structure called quadtree proposed by S. L. Horowitz and S. Pavlidis [6] in the context of image segmentation grayscale.

The quadtree is a tetra-tree in which each node has exactly four son nodes, except the terminal nodes. Each node corresponds to a block that is an area of the image of square shape, the number of rows and columns which is a power of two. The root of the tree corresponds to the entire image must be square and the number of rows and columns is also a power of two (fig.4).

Each block with a quadtree node of the initial partition is recursively analyzed to determine whether it should be divided 
into four sub-blocks. The recursive analysis stops when each sub-block follows a predicate homogeneity.

Each block with a quadtree node of the initial partition is recursively analyzed to determine whether it should be divided into four sub-blocks. The recursive analysis stops when each sub-block follows a predicate homogeneity.

At this stage of the analysis, some adjacent blocks in the image are the same color characteristics. This is why these pairs of blocks are merged. The analysis stops when there is more torque that respects the predicate fusion.

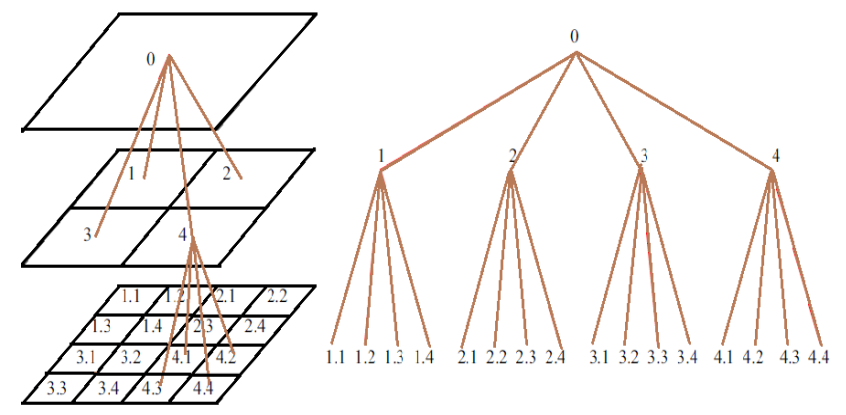

Fig. 4. Structure pyramidal quadtree

The major drawback of the quadtree is the rigidity of the square cut. It leads to a partitioning of the global image that does not always form regions present in the image. In addition, the consolidation phase of the blocks is sensitive to the order of the path quadtree.

\section{b) Voronoi diagram [7]}

The Voronoi diagram approach is part of an iterative process of divisions, mergers and can be seen as an improvement of the segmentation analysis of a quadtree. Phase division is not carried out by cutting square-shaped regions, but by cutting Voronoi polygons that fit the shapes of these regions in the image.

The Voronoi diagram generates a partition of the image from germs. Each seed is assigned a Voronoi region consists of all of the closest seed pixels.

Fig.4 shows an example of a Voronoi diagram. In this figure, the seeds are the red dots. The closest a seed pixels are those listed in the blue centered on the seed polygon.

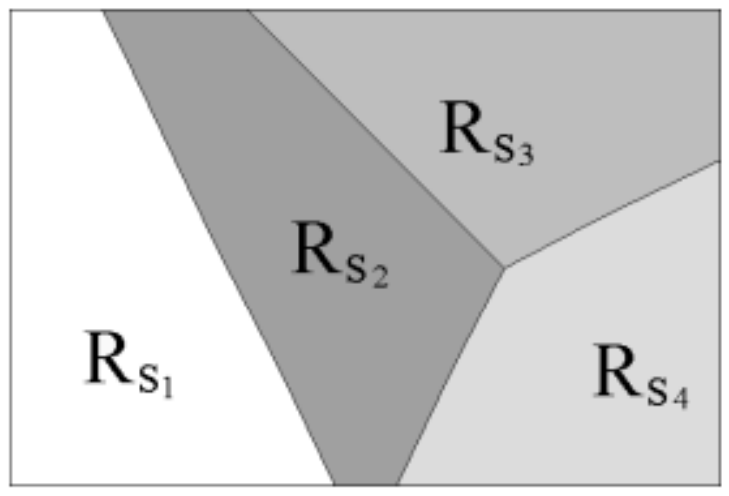

Fig. 5. Example of Voronoi diagram [7].
This approach is presented for grayscale images, but can be generalized to color images.

The given segmentation method comprises an initialization step, a division step followed by a melting step:

- Initialization: Germs are positioned and uniformly distributed in the image using a Poisson process. Each seed is associated with a region whose boundaries are established using a Voronoi diagram.

- Division: A predicate homogeneity is calculated for each region and the non-homogeneous regions are divided by introduction of new seeds. The Voronoi diagram is updated and the division process is repeated until all the Voronoi regions meet a predicate homogeneity.

- Melting: Germs that do not correspond to an object in the image are eliminated in this final step. Thus, adjacent regions whose average colors are close and where the length of the border divided by the sum of their perimeters is less than a threshold are merged.

\section{Segmentation analysis of pixels}

This second part is mainly devoted to methods from the field of classification of multidimensional data that are applied to the segmentation of color images.

These methods consider a region as a set of connected pixels belonging to the same class. They therefore assume that the pixels that belong to the same region have similar colors and form a cloud of points clearly identifiable in the representation space $3 \mathrm{D}$ color. The classification consists in finding these clouds of points that correspond to the classes of pixels present in the image.

Supervised classification is called when a priori information is introduced into the process of building classes as a learning sample. Classification is called unsupervised when no a priori knowledge is available. However, we can question the validity of the so-called unsupervised segmentation in the sense that the methods generally involve thresholds that introduce certain way information on the classes of pixels to build.

In this section, we present two families of methods:

- Unsupervised pixel classification

- unsupervised methods

\section{1) Unsupervised pixel classification}

\section{a) K-means algorithm}

The k-means clustering algorithm [8] is the best known and most widely used because of its simplicity of implementation. It partitions the image data into $\mathrm{K}$ clusters.

Unlike other methods known hierarchical structure that create a "cluster tree" to describe groups, k-means creates only one level of clusters.

The algorithm returns a data partition in which objects within each cluster are as close as possible to each other and as far as possible objects of other clusters. Each cluster in the partition is defined by its objects and its centroid. 
The k-means is an iterative algorithm that minimizes the sum of distances between each object and the centroid of the cluster.

The initial position of the centroid determines the final result, so that the centroid should be initially placed as far as possible from each other so as to optimize the algorithm. Kmeans cluster objects exchange until the sum can no longer decreasing. The result is a set of compact and clearly separated clusters, provided that they have chosen the correct value of the number of clusters $\mathrm{K}$.

The K-means algorithm is an iterative technique that is used to partition an image into K clusters. The basic algorithm is:

1) Pick K cluster centers, either randomly or based on some heuristic

2) Assign each pixel in the image to the cluster that minimizes the distance between the pixel and the cluster center

3) Re-compute the cluster centers by averaging all of the pixels in the cluster

4) Repeat steps 2 and 3 until convergence is attained

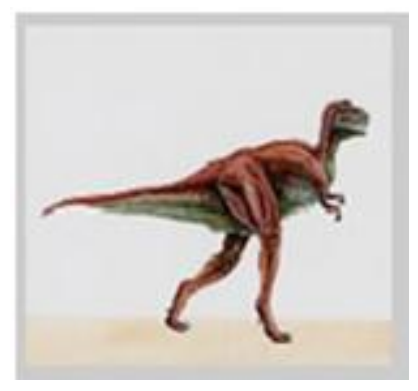

(1)

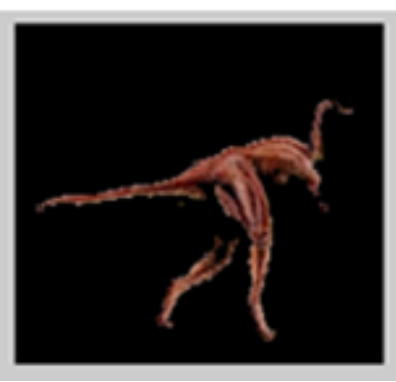

(2)
Fig. 6. (1) Original image, (2) Image segmented.

a) Fuzzy c-means

The algorithm of fuzzy c-means [9] is a fuzzy clustering algorithm based on optimization of a quadratic criterion of classification where each class is represented by its center of gravity. The algorithm requires knowing the number of classes in advance and generates classes so that the sum of squared deviations interclass and intraclass are respectively maximum and minimum.

Y. W. Lim and S. U. Lee [10] proposed to segment color images in a coarse to fine decomposition by performing a first segmentation multithresholding, followed by a more detailed segmentation by applying an algorithm of fuzzy c-means.

They analyze marginally each color component by the scale space filtering approach to determine the thresholds that separate modes detected. This technique is proposed by Witkin in [11] and to determine the most prominent peaks dimensional histograms. Thus, the color space is partitioned into subspaces defined by different thresholds determined for each component. These subspaces correspond to each class of pixels. Among these classes, some of which are composed of a number of pixels too low to be meaningful. They are then removed. The fine segmentation is to classify the unlabeled pixels by the coarse segmentation. Thus, the pixels that do not belong to any class are assigned to the class for which their degree is maximum.

R. Krishna Priya, C. Thangaraj and C. Kesavadas [12] propose to improve the classical algorithm of fuzzy c-means taking into account the local spatial information. Thus, the criterion to be minimized is modified by inserting a weighting which depends on the color difference between the central pixel and its neighboring pixels. Taking into account the local spatial information improves the classification results and provides satisfactory segmentation results.

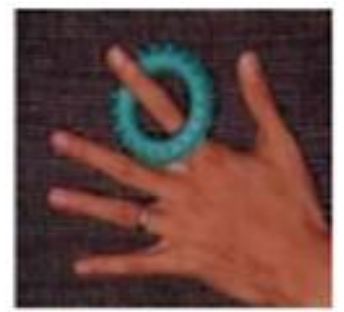

(a)

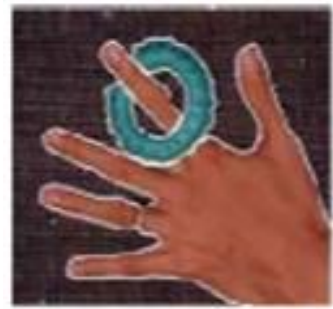

(b)
Fig. 7. (a) Test color image, (2) Image segmented [12].

\section{b) Fisher algorithm}

Fisher algorithm [13] is to perform a classification of pixels of the image into $\mathrm{k}$ classes, using the partitioning of a histogram grayscale $\mathrm{k}$ disjoint classes such that the sum of the variances of classes is minimal.

Under the color should be applied this algorithm separately the three components of the color space representation.

\section{2) Supervised pixel classification}

\section{a) Mono-dimensional histogram}

Many authors define classes of pixels per recursive analysis of one-dimensional histograms of different color components to segment images by techniques multithresholding [14].

The histograms dimensional recursive analysis can highlight, as and when the iterations, the modes corresponding to the classes of pixels. It partitions the set of pixels of the image into different classes according to the following algorithm:

Stacking all the pixels of the image in the stack of pixels to be processed.

While the stack of the pixels to be processed is non-empty do

Unstack the pixels,

Calculate dimensional histogram of these pixels,

Search the histogram shows how the most important

If the mode is large enough then

Constitute a new class with the pixels that belong to this mode,

Stacking the pixels not belonging to this user in the stack of the pixels to be processed, 


\section{End If}

\section{End while}

The methods proposed in literature differ in:

- The choice of the color components used

- The method and criteria for extracting modes.

- The determination of the most representative component.

- The criteria for stopping the recursive analysis of histograms

\section{b) Nearest neighbor algorithm}

The method of k-nearest neighbors (k-NN)[15] is a method for estimating non-parametric density [15]. It is to seek from a learning base and a distance set to "data space" K-nearest neighbors of an element. That is to calculate the estimate of density $r i$ of the class $\mathrm{C}_{\mathrm{i}}$ at the point $x$ defined by the following relationship:

$$
r i(x)=\frac{K i(x)}{n i \times V(x)}
$$

Where:

$K i(x)$ : is the number of points $\mathrm{C}_{\mathrm{i}}$ belonging to k-nearest neighbor the $x$.

$n i$ : is the cardinality of the class $\mathrm{C}_{\mathrm{i}}$.

$V(x)$ : Volume of the smaller ball containing k-nearest neighbor the $\mathrm{x}$.

The probability of belonging to a class of the element $\mathrm{x}$ is proportional to the number of examples of this class among its $\mathrm{k}$-nearest neighbors. The class of $\mathrm{x}$ is then the element having the highest probability.

\section{EDGE DETECTION APPROACH}

Early approaches to contour detection aim at quantifying the presence of a boundary at a given image location through local measurements. The Roberts [16], Sobel [17], and Prewitt [18] operators detect edges by convolving a grayscale image with local derivative filters. Marr and Hildreth [19] use zero crossings of the Laplacian of Gaussian operator. The Canny detector [20] also models edges as sharp discontinuities in the brightness channel, adding non-maximum suppression and hysteresis thresholding steps. A richer description can be obtained by considering the response of the image to a family of filters of different scales and orientations. An example is the Oriented Energy approach, which uses quadrature pairs of even and odd symmetric filters. Lindeberg [21] proposes a filterbased method with an automatic scale selection mechanism.

More recent local approaches take into account color and texture information and make use of learning techniques for cue combination. Martin et al. [22] define gradient operators for brightness, color, and texture channels, and use them as input to a logistic regression classifier for predicting edge strength. Rather than rely on such hand-crafted features, Dollar et al. [23] propose a Boosted Edge Learning (BEL) algorithm which attempts to learn an edge classifier in the form of a probabilistic boosting tree from thousands of simple features computed on image patches. An advantage of this approach is that it may be possible to handle cues such as parallelism and completion in the initial classification stage. Mairal et al. [24] create both generic and class-specific edge detectors by learning discriminative sparse representations of local image patches. For each class, they learn a discriminative dictionary and use the reconstruction error obtained with each dictionary as feature input to a final classifier. 


\section{Advantages and Disadvantages of Segmentation Technique}

\begin{tabular}{|c|c|c|}
\hline $\begin{array}{c}\text { Segmentation } \\
\text { Technique }\end{array}$ & Advantages & Disadvantages \\
\hline Regions dividing & $\begin{array}{l}\text { - Guaranteed to produce coherent regions. } \\
\text { - Works from the inside out instead of the } \\
\text { outside inwhich object a pixel belongs to is } \\
\text { immediate. }\end{array}$ & $\begin{array}{l}\text { - Cutting areas unfaithful to the original } \\
\text { image }\end{array}$ \\
\hline Region Growing & $\begin{array}{l}\text { - Simple and fast. } \\
\text { - It allows the object segmentation complex } \\
\text { topology. } \\
\text { - It preserves the shape of each region of the } \\
\text { image. }\end{array}$ & $\begin{array}{l}\text { - The computation is consuming, no matter } \\
\text { the time or power. } \\
\text { - Noise or variation of intensity may result } \\
\text { in holes or oversegmentation. } \\
\text { - This method may not distinguish the } \\
\text { shading of the real images. }\end{array}$ \\
\hline Region Merging & $\begin{array}{l}\text { - The image could be split progressively } \\
\text { according to our demanded resolution because } \\
\text { the number of splitting level is determined by } \\
\text { us. } \\
\text { - We could split the image using the criteria we } \\
\text { decide, such as mean or variance of segment } \\
\text { pixel value. In addition, the merging criteria } \\
\text { could be different to the splitting criteria }\end{array}$ & $\begin{array}{l}\text { - This method depends on the merging } \\
\text { criterion that can influence the final result } \\
\text { segmentation. } \\
\text { - It can introduce the effect of sub- } \\
\text { segmentation. }\end{array}$ \\
\hline Quadtree & $\begin{array}{l}\text { - The quadtree structure is very common due to } \\
\text { its simplicity and data secondly its low } \\
\text { computation time. }\end{array}$ & $\begin{array}{l}\text { - The rigidity of the square cut } \\
\text { - It leads to a partitioning of the overall } \\
\text { image does not always respect the form } \\
\text { regions present in the image. } \\
\text { - The division quadtree generally provides } \\
\text { an over-segmentation }\end{array}$ \\
\hline Voronoi diagram & $\begin{array}{l}\text { - The based on the Voronoi diagram approach } \\
\text { can be considered a improved segmentation } \\
\text { analysis of quad-tree. } \\
\text { - Voronoi fit the shapes of regions present in } \\
\text { the image. }\end{array}$ & $\begin{array}{l}\text { - Initialization germs can lead to different } \\
\text { segmentation results. }\end{array}$ \\
\hline K-means & $\begin{array}{l}\text { - K-means algorithm is easy to implement. } \\
\text { - Its time complexity is } \mathrm{O}(\mathrm{n}) \text {, where } \mathrm{n} \text { is the } \\
\text { number of patterns. It is faster than the } \\
\text { hierarchical clustering. }\end{array}$ & $\begin{array}{l}\text { - The result is sensitive to the selection of } \\
\text { the initial random centroids. } \\
\text { - We cannot show the clustering details as } \\
\text { hierarchical clustering does. }\end{array}$ \\
\hline $\begin{array}{l}\text { Histogram } \\
\text { Thresholding }\end{array}$ & $\begin{array}{l}\text { - It does not need a prior information of the } \\
\text { image. And it has less computational } \\
\text { complexity }\end{array}$ & $\begin{array}{l}\text { - Does not work well for an image without } \\
\text { any obvious peaks or with broad and flat } \\
\text { valleys } \\
\text { - Does not consider the spatial details, so } \\
\text { cannot guarantee that the segmented } \\
\text { regions are contiguous }\end{array}$ \\
\hline
\end{tabular}




\section{CONCLUSIONS}

In this study, the overview of various segmentation methodologies applied for digital image processing is explained briefly. The study also reviews the research on various research methodologies applied for image segmentation and various research issues in this field of study. This study aims to provide a simple guide to the researcher for those carried out their research study in the image segmentation.

\section{REFERENCES}

[1] H. D. Cheng, X. H. Jiang, Y. Sun, et J. Wang. Color image segmentation : advances and projects. Pattern Recognition, 34 :2259-2281, 2001

[2] Slawo Wesolkowski ; Paul W. Fieguth. Color image segmentation using vector angle-based region growing. SPIE.4421..910W, 2002.

[3] R. Schettini. A segmentation algorithm for color images. Pattern Recognition Letters, 14 :499-506, 1993.

[4] J. Stawiaski and E. Decenciere. Region Merging via Graph-Cuts. ICS XII - 2007: International Congress of the International Society of Stereology, Saint-Etienne, France.

[5] V. Coutance. La couleur en vision par ordinateur. Application à la robotique. Thèse de doctorat, Université Paul Sabatier de Toulouse, Janvier 2001.

[6] S. L. Horowitz et S. Pavlidis. Picture segmentation by a directed split and merge procedure.In 2nd Int. Joint Conf. on Pattern Recognition, pages 424-433, 1991.

[7] Andres Arbelaez, P.; Cohen, L.D. Color Image Segmentation by Voronoi Partitions. 2004.

[8] Anil Z Chitade, Dr. S.K. Katiyar, "Color Based Image Segmentation using K-Means Clustering", International Journal of Engineering Science and Technology Vol. 2(10), 2010, 5319-5325

[9] J. C. Bezdeck. Pattern recognition with fuzzy objective function algorithms. Plenum Press Ed., New York, 1997.

[10] Y. W. Lim et S. U. Lee. On the color image segmentation algorithm based on the thresholding and the fuzzy c-means techniques. Pattern Recognition, 23(9) :935-952, 1995.
[11] A.W.C. Liew, S.H. Leung, et W.H. Lau. Fuzzy image clustering incorporating spatial continuity. IEE Proceedings on Vision Image Signal Processing, 147(2) :185-192, April 2000.

[12] R. Krishna Priya1, C. Thangaraj and C. Kesavadas. Fuzzy C-Means method for Colour Image Segmentation. ISSN (Online): 1694-0814. 2011

[13] W. FISHER. « On grouping for maximum homogeneity ». JASA, vol. 53, pages 789-798, 1998.

[14] R. DUDA, P.HART \& D.STORK. « Pattern Classification ». Xiley Interscience- 2e édition, 2001.

[15] Wojciech Bieniecki and Szymon Grabowski, "Nearest Neighbor

Classifiers for Color Image Segmentation", TCSET' 2004 Feb 24-28

[16] L. G. Roberts, "Machine perception of three-dimensional solids," In Optical and Electro-Optical Information Processing, J. T. Tippett et al. Eds. Cambridge, MA: MIT Press, 1965.

[17] R. O. Duda and P. E. Hart, Pattern Classification and Scene Analysis. New York: Wiley, 1973.

[18] J. M. S. Prewitt, "Object enhancement and extraction," In Picture Processing and Psychopictorics, B. Lipkin and A. Rosenfeld. Eds.

[19] D. C. Marr and E. Hildreth, "Theory of edge detection," Proceedings of the Royal Society of London, 1980.

[20] J. Canny, “A computational approach to edge detection," PAMI, 1996.

[21] T. Lindeberg, "Edge detection and ridge detection with automaticscale selection," IJCV, 1998.

[22] D. Martin, C. Fowlkes, and J. Malik, "Learning to detect natural image boundaries using local brightness, color and texture cues,"

[23] P. Dollar, Z. Tu, and S. Belongie, "Supervised learning of edges and object boundaries," CVPR, 2006.

[24] J. Mairal, M. Leordeanu, F. Bach, M. Hebert, and J. Ponce, "Discriminative sparse image models for class-specific edge detection and image interpretation," ECCV, 2008. 